\title{
Heavy flavour physics in heavy ion collisions at LHC
}

\section{Giuseppe Eugenio Bruno*}

Dipartimento Interateneo di Fisica “M. Merlin” and Sezione INFN, Bari, Italy

E-mail: giuseppe.bruno@ba.infn.it

Heavy ion collisions at the LHC energies are used to study the physics of strongly interacting matter at very high energy densities, which exceed by one order of magnitude the critical value predicted by lattice QCD for a phase transition into a quark gluon plasma. Heavy flavour quarks, namely charm and beauty, are produced in hard parton scattering processes in the early time of the collisions and thus they experience the full evolution of the system. Hadrons with open or hidden heavy flavour are thus ideal probes to study the properties of the strongly interacting matter produced in heavy ion collisions at the LHC. In this conference contribution I have reviewed recent results and discussed the prospects for the future.

The International Conference on B-Physics at Frontier Machines - BEAUTY2018

6-11 May, 2018

La Biodola, Elba Island, Italy

${ }^{*}$ Speaker. 


\section{Introduction to ultra-relativistic heavy ion collisions}

Lattice QCD calculations indicate that, at a critical temperature around $160 \mathrm{MeV}$, strongly interacting matter undergoes a phase transition to a state where the quarks and gluons are no longer confined into hadrons, the Quark Gluon Plasma (QGP). In Pb-Pb collisions at the LHC energies, a QGP with almost null baryo-chemical potential and high temperature is created, similar to the state of the early Universe a few microseconds after the Big Bang. The system produced in the collisions evolve with time: after about $0.5 \mathrm{fm} / c$ (pre-equilibrium phase) a thermal local equilibrium is reached. The QGP then experiences a volume expansion for several $\mathrm{fm} / c$, which can be described by relativistic (viscous-)hydrodynamics, while cooling down until the temperature becomes smaller than the critical value and the system undergoes the transition into the hadronic phase. At this stage the system can still change its chemical composition, while experiencing a further expansion and cooling in the hadronic (or mixed) phase. At the "chemical freeze-out" the relative abundances of different particle species are fixed. Afterwards, there can still be an expansion phase where elastic collisions maintain the thermal equilibrium, until the particles decouple and freely stream in the vacuum (the so called "thermal" or "kinetic" freeze-out, when the momentum distributions of the particles are fixed). The above picture has emerged from a detailed study of several observables, performed with the first LHC run and previously at lower energy at RHIC, mainly in the light flavour sector (see [1,2] for reviews and [3] for a collection of recent results and theoretical developments). One striking feature of heavy-ion collisions is the anisotropic particle flow [4]. It arises from initial collision geometry anisotropies being converted by the pressure gradients of the QGP medium to final-state particle transverse momentum anisotropies. The anisotropic flow is described by the coefficient $v_{\mathrm{n}}$ of a Fourier series decomposition of the azimuthal distribution of the produced particles, $\mathrm{d} N / \mathrm{d} \phi \propto 1+2 \sum_{\mathrm{n}=1}^{\infty} v_{\mathrm{n}} \cos \mathrm{n}\left(\phi-\Psi_{\mathrm{n}}\right)$. The "jet quenching" is a second most striking effect observed in heavy ion collisions at high energies. High $p_{\mathrm{T}}$ partons, produced in hard scattering processes, loose a significant fraction of their energies while traversing the medium. Experimentally the most simple observable related to this effect is the suppression, at $p_{\mathrm{T}}$ above a few $\mathrm{GeV} / c$, in the production of the leading hadrons with respect to proton-proton collisions scaled by the average number of binary nucleon-nucleon collisions $\left(N_{\text {bin }}\right)$ expected in nucleus-nucleus collisions. The nuclear modification factor, which is defined as $R_{\mathrm{AA}}=\mathrm{d} N^{\mathrm{A}-\mathrm{A}} / \mathrm{d} p_{\mathrm{T}} /\left(N_{\mathrm{bin}} \mathrm{d} N^{\mathrm{pp}} / \mathrm{d} p_{\mathrm{T}}\right)$, quantifies this effect. Figure 1 shows $R_{\mathrm{AA}}$ versus $p_{\mathrm{T}}$ for charged particles in $\mathrm{Pb}-\mathrm{Pb}$ collisions and in $\mathrm{pPb}$ collisions (the symbol $R_{\mathrm{pA}}$ is used in this case), and for particles that do not interact strongly (photons and electro-weak bosons). A suppression up to a factor 5 is observed for charged particles in central $\mathrm{Pb}-\mathrm{Pb}$ collisions, while no significant modifications are observed in $\mathrm{pPb}$ collisions or for particles without colour charge.

\subsection{Heavy Flavour probes of the QGP}

Independently of their transverse momentum, heavy quarks are produced at the initial stage of the collision in high-virtuality scattering processes. They interact with the medium and experience its full evolution since the early phase of the collision. Open charm and beauty hadrons with $p_{\mathrm{T}}$ above a few $\mathrm{GeV} / c$ are ideal probes to test and constrain the mechanism of in-medium energy loss, which is sensitive to the type (quark vs. gluon) and mass of the parton (charm vs. beauty). At 


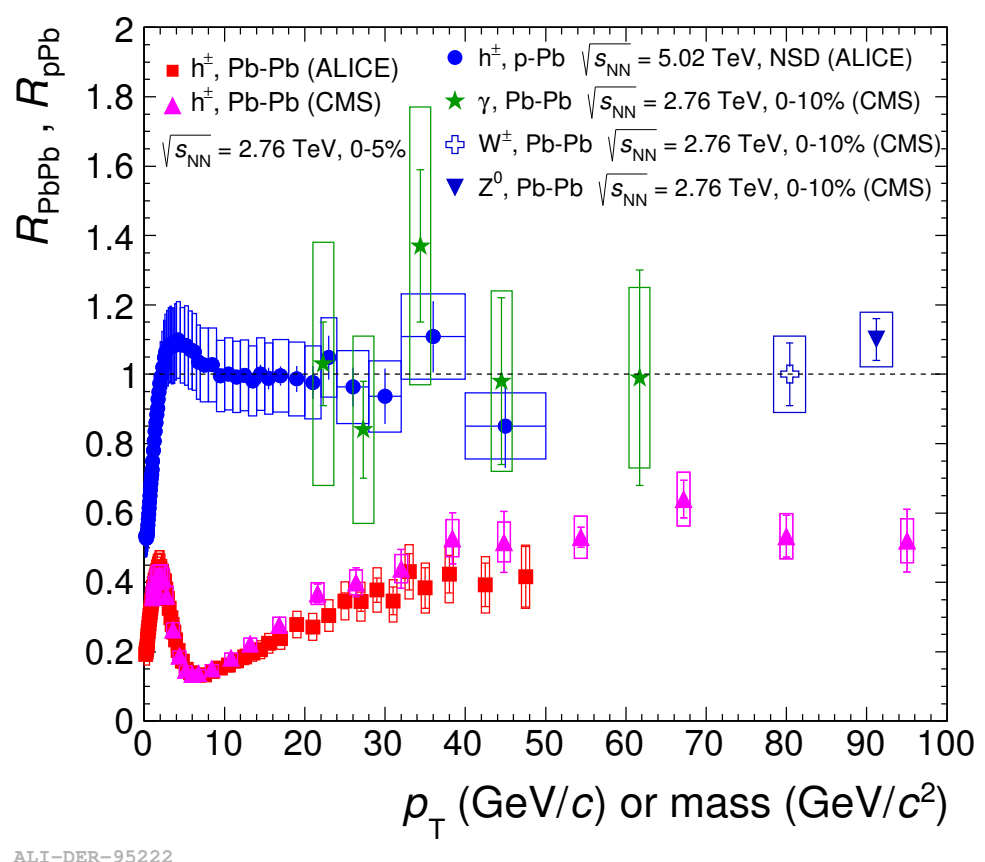

Figure 1: The nuclear modification factor of charged particles $\left(\mathrm{h}^{ \pm}\right)$and of photons versus transverse momentum and for electroweak bosons versus mass measured in $\mathrm{Pb}-\mathrm{Pb}$ collisions at $\sqrt{s_{\mathrm{NN}}}=2.76 \mathrm{TeV}$ and for $\mathrm{h}^{ \pm}$in $\mathrm{pPb}$ collisions at $\sqrt{s_{\mathrm{NN}}}=5.02 \mathrm{TeV}[5]$.

lower $p_{\mathrm{T}}$ open heavy flavoured hadron production provide information about the thermalization of partons in the QGP. Heavy-quark elliptic flow is especially sensitive to the partonic equation of state. In the hidden heavy quark sector, the quarkonium dissociation in the QGP and, in the

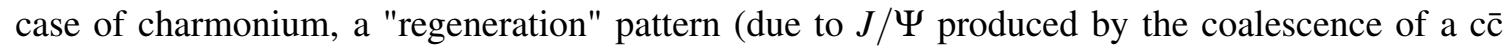
quark pair from the medium), are considered as ideal probes of deconfinement and of the medium temperature.

\section{Recent $\mathrm{Pb}-\mathrm{Pb}$ results: a selection}

Indication of a mass dependent suppression, i.e $R_{\mathrm{AA}}(b)>R_{\mathrm{AA}}(c)$ has been obtained with run1 data from LHC [6], by the comparison of the ALICE D meson and the CMS non-prompt $J / \psi$, coming from the decay of beauty hadrons. Figure 2 (top panel) shows this comparison versus the centrality of the $\mathrm{Pb}-\mathrm{Pb}$ collisions. The $p_{\mathrm{T}}$ ranges of $\mathrm{D}$ mesons and of non-prompt $J / \psi$, in this comparison, have been chosen to match a similar $p_{\mathrm{T}}$ range for the parent beauty hadrons as for the D mesons. More precise results from run2 data of the LHC are confirming these findings [7] (see bottom panels of Figure 2).

In the light favour sector, precise measurements of the $v_{2}$ coefficients for different particle types, along with those of higher coefficients (not discussed at the Conference), have been accomplished. The observed mass ordering for $p_{\mathrm{T}}<2 \mathrm{GeV} / c$ (see top panel of Figure 3 ) is described by hydrodynamical models for a medium that behaves as an almost ideal fluid (very small viscosity). The very precise run 2 data, in particular for the $\phi$ meson, have revealed a baryon versus meson 

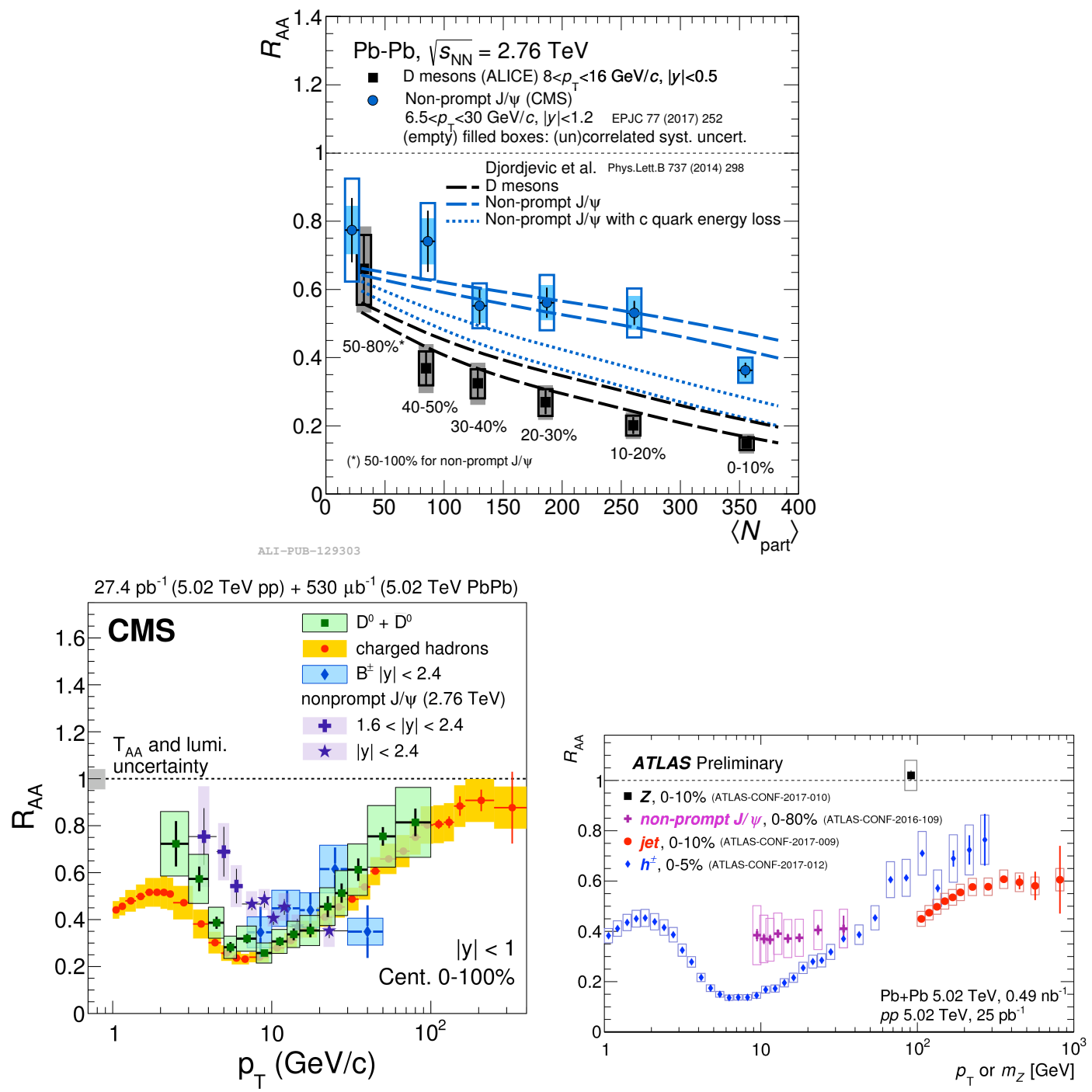

Figure 2: Top panel: $R_{\mathrm{AA}}$ as a function of centrality, measured in terms of nucleons participating to the collisions for D mesons (ALICE) and for non-prompt $J / \psi$ coming from the decay of B hadrons (CMS). Bottom panels: $R_{\mathrm{AA}}$ as a function of $p_{\mathrm{T}}$ for different particles as measured by CMS and ATLAS.

grouping at higher $p_{\mathrm{T}}$ (in the range $2-6 \mathrm{GeV} / c$ ), which is suggestive of collective flow well developed at the quark-level and a sizeable recombination mechanism in this $p_{\mathrm{T}}$ range from the quarks of the flowing medium. In the heavy flavour sector there is evidence for charm flowing with the medium, but the $\mathrm{D}$ meson $v_{2}$ is smaller than that of pions $[8,9]$ (see middle and bottom panels of figure 3). The CMS experiment has also reported a D meson measurement of the $v_{3}$ coefficient [9], which is sensitive to the initial geometry fluctuations and to the interaction strength between charm quarks and the medium. The simultaneous description of the energy loss $\left(R_{\mathrm{AA}}\right)$ and of the elliptic flow $\left(v_{2}\right)$ for heavy flavour particles poses severe constraints to theoretical models. Figure 4 shows a compilation of theoretical models with superimposed ALICE results in $\mathrm{Pb}-\mathrm{Pb}$ collisions at $5.02 \mathrm{TeV}$ [10]. The models where charm quarks pick up collective flow via recombination and/or subsequent elastic collisions in the expanding medium do better at describing both observables, 

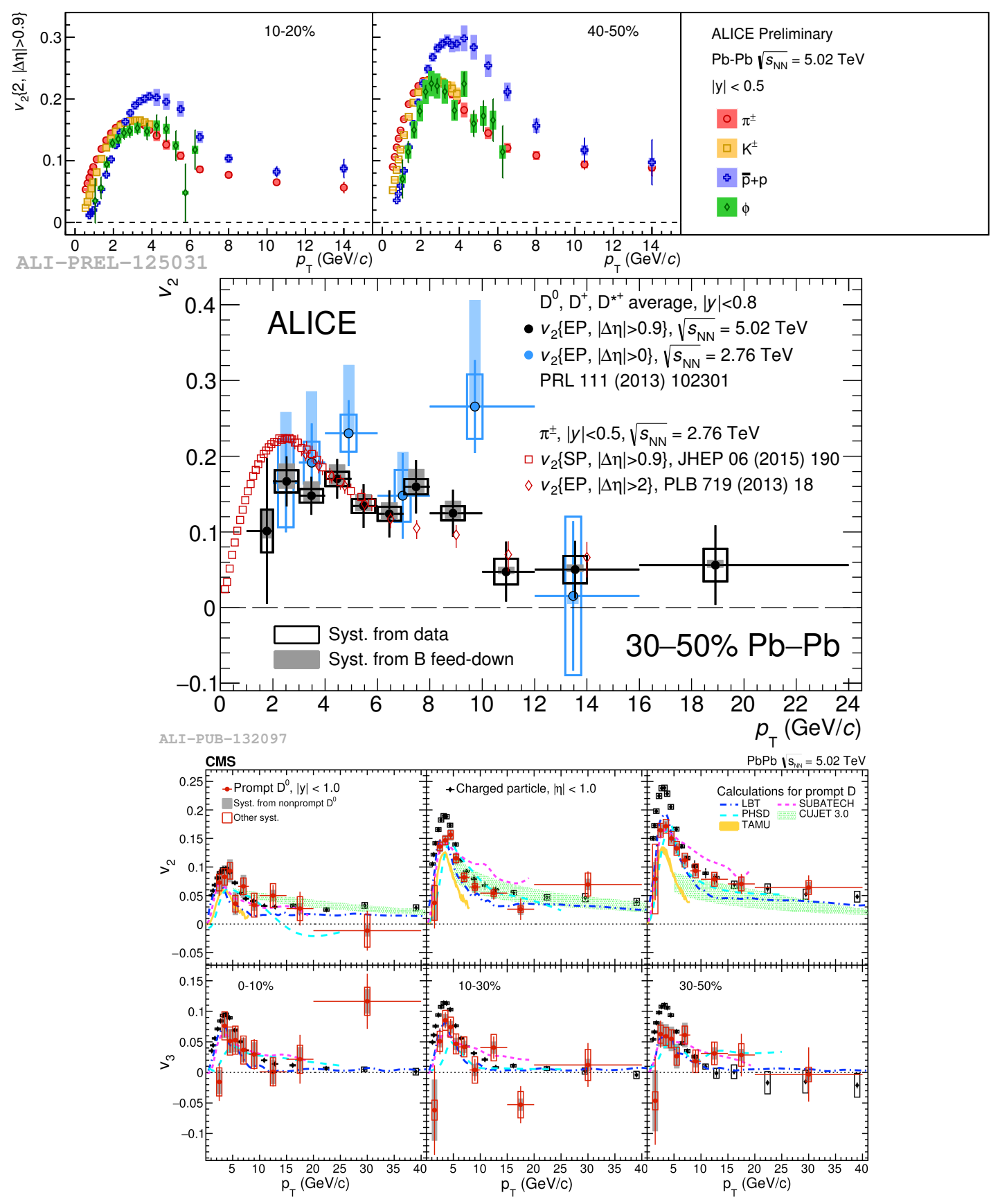

Figure 3: The $v_{2}$ coefficient versus $p_{\mathrm{T}}$ for light particles (top panels) and for $\mathrm{D}$ mesons as measured by ALICE (middle panel) and CMS (bottom panels) in $\mathrm{Pb}-\mathrm{Pb}$ collisions at the LHC. CMS has also reported results for the $3^{\text {rd }}$ harmonic coefficient $v_{3}$ that quantifies the "triangular flow". 
see [10] for a detailed discussion.
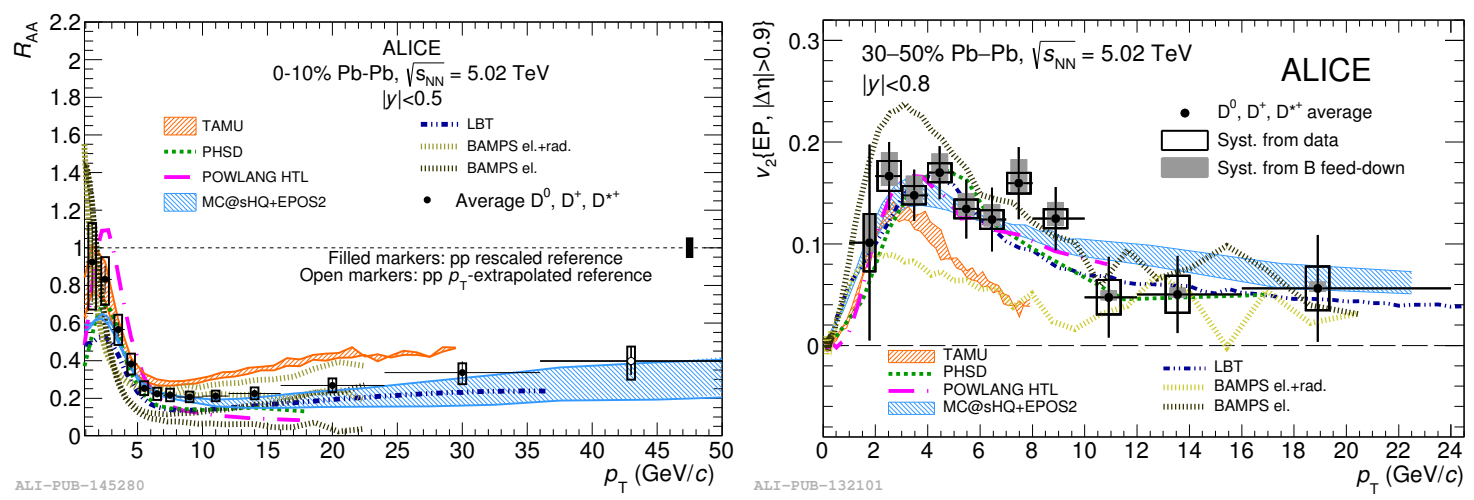

Figure 4: $R_{\mathrm{AA}}$ (left) and $v_{2}$ (right) versus $p_{\mathrm{T}}$ measured by ALICE as compared to theoretical models.

While the energy loss (i.e. the $R_{\mathrm{AA}}$ factor) of beauty has been investigated already with good precisions at intermediate and high $p_{\mathrm{T}}$, it remains to be studied in the low $p_{\mathrm{T}}$ region. The study of the collectivity ( $v_{2}$ and higher $v_{n}$ coefficient) for beauty could be accomplished only with LHC run3 data.

At the Conference, I have also discussed the hadronisation mechanism in the open charm sector. Preliminary results for the $\Lambda_{c}$ baryon and published results for the $D_{\mathrm{s}}$ meson [10] indicate similarities with the light sector: enhancement of the baryon over meson ratio with respect to $\mathrm{pp}$ collisions and enhancement in the production of particles with strangeness content. Both observations are justified in a scenario where hadrons are formed by recombination of quarks.

In the quarkonium sector, the LHC has provided since the first run two spectacular results: a much smaller suppression at low $p_{\mathrm{T}}$ of $J / \psi$ with respect to RICH energy [11], which was predicted as a signature for the recombination scenario, and the sequential dissociation of the $\Upsilon$ states [12]. Run2 data have confirmed these findings and have allowed a significant reduction of the experimental uncertainties [13, 14]. Figure 5 shows the invariant mass distribution of muon pairs measured by CMS (left panel) and the level of suppression of the $\Upsilon(2 \mathrm{~S})$ state with respect to $\Upsilon(1 \mathrm{~S})$ ground state in $\mathrm{Pb}-\mathrm{Pb}$ collisions at $\sqrt{s_{\mathrm{NN}}}=5.02 \mathrm{TeV}$. Moreover, double differential studies have become affordable, which in particular have allowed to observe a non-null elliptic flow for the $J / \psi[15]$ : given the small interaction cross-section of the $\mathrm{J} / \psi$ meson with hadrons, this result further corroborates the recombination scenario where the flow of $\mathrm{J} / \psi$ is inherited from that of $c$ and $\bar{c}$ quarks.

\section{The intriguing "small systems"}

The standard "paradigm" of ultra-relativistic heavy ion collision assigns the role of "control experiments" to the $\mathrm{pp}$ and $\mathrm{p}-\mathrm{Pb}$ systems. Indeed the results discussed so far are mainly based on the comparison between the results in the $\mathrm{Pb}-\mathrm{Pb}$ systems and those in minimum-bias $\mathrm{pp}$ and $\mathrm{pPb}$ (the latter to study the initial state or "cold-nuclear matter" effects). However, in a small sub-sample of both $\mathrm{pp}$ and $\mathrm{pPb}$ events, obtained by selecting a tiny fraction of events, corresponding to those 

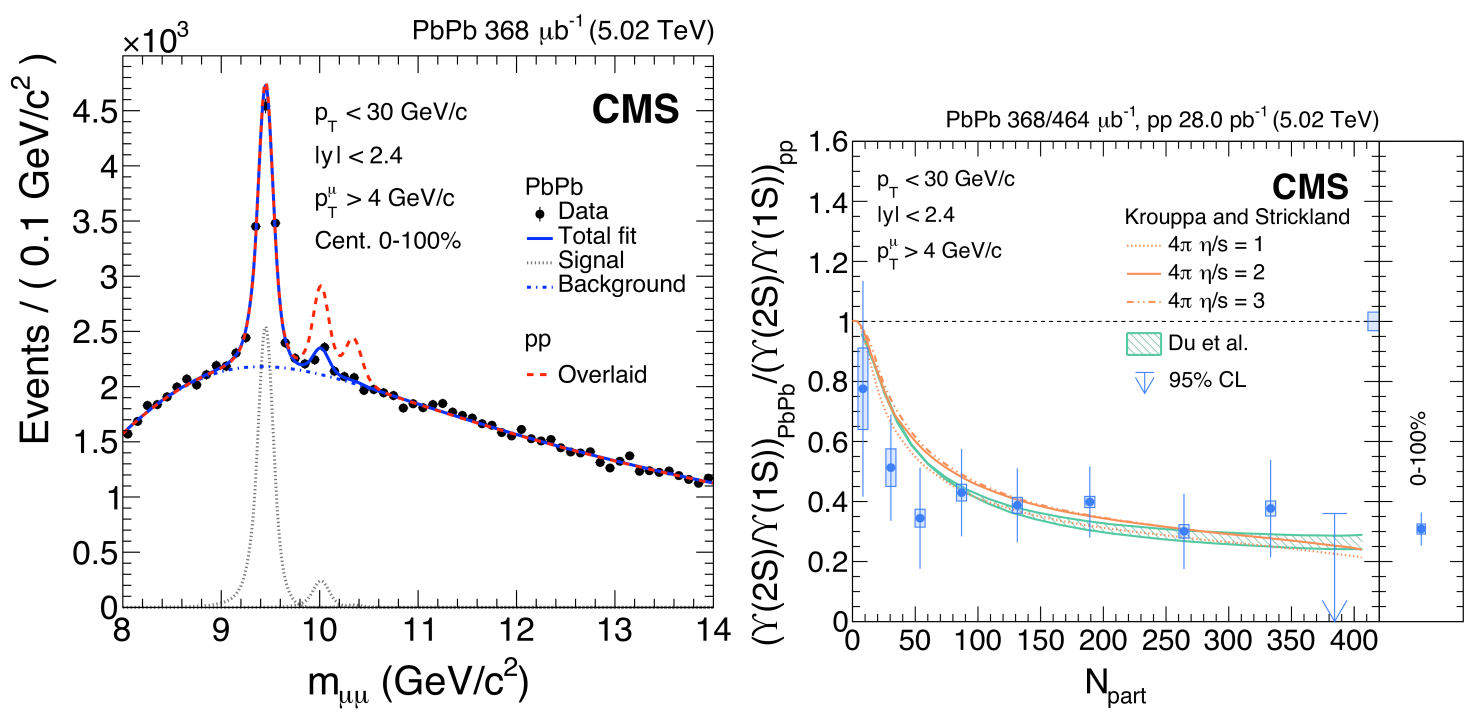

Figure 5: Left panel: the invariant mass distribution of muon pairs measured by $\mathrm{CMS}$ in $\mathrm{Pb}-\mathrm{Pb}$ collisions (data points) and in pp collisions at the same energy (dotted red line). Right panel: double ratio of the $\Upsilon(2 \mathrm{~S})$ to the $\Upsilon(1 \mathrm{~S})$ yield in $\mathrm{Pb}-\mathrm{Pb}$ collisions to that in pp collisions as a function of the centrality of the $\mathrm{Pb}-\mathrm{Pb}$ collisions.

with the highest event activity (i.e. the highest multiplicity of charged tracks), striking properties are observed that resemble those due to collectivity or QGP-like properties of the $\mathrm{Pb}-\mathrm{Pb}$ system:

- the azimuthal modulation of the particle production, that could signal the onset of a collective expansion [16];

- a baryon to meson ratio much larger than in minimum-bias pp collisions that increases with multiplicity [17];

- a reduction of the relative yields of excited over ground quarkonium states [18];

- an enhancement in the production of strange particles [19].

No signal of energy loss in these events is observed, within the present uncertainties. Recent results have provided indication of collectivity also for open heavy flavour and charmonium [20, 21, 22]. Figure 6 shows the $v_{2}$ coefficient measured for D mesons as compared to strange particles and for leptons coming from open heavy flavour decays.

\section{Summary and outlook}

The LHC is already providing precise measurements in the heavy flavour sector, that allow us to pose stringent constraints on the models describing the properties of the system (e.g., the transport coefficients, the viscosity over entropy-density ratio) and its dynamical evolution. A number of open questions remain to be answered: are charm quarks fully thermalized in the plasma? Do also beauty quark take part to the collective dynamics? How relevant is the recombination for beauty?

"Small systems", i.e. pp and $\mathrm{pPb}$ collisions with very high event activity, have become a new laboratory to study QCD in extreme conditions. One can start to ask how small can be a droplet of 

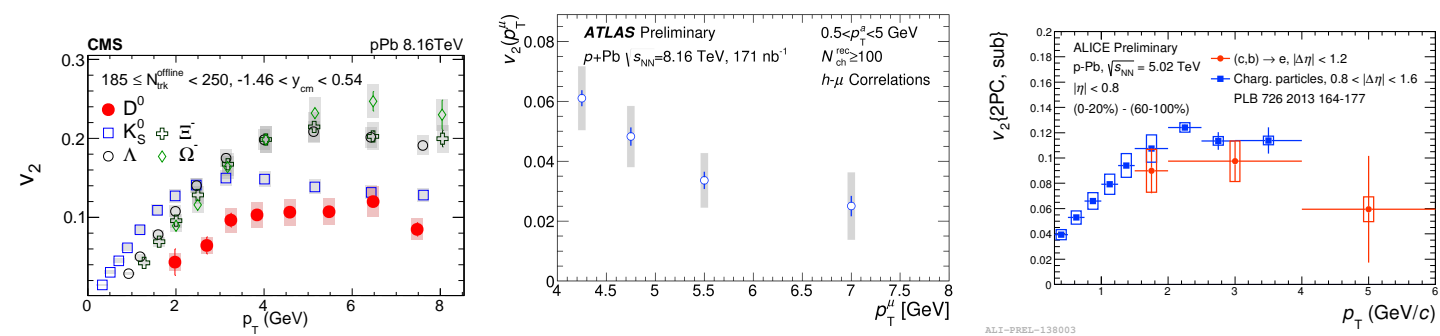

Figure 6: The $v_{2}$ coefficient measured in high multiplicity $\mathrm{pPb}$ collisions versus $p_{\mathrm{T}}$ for $\mathrm{D}$ mesons as compared to strange particles (left panel), for muons and electrons coming from heavy-flavour decays (middle and right panel, respectively).

QGP and what are its properties. Thanks to the detector upgrades and with the next runs (run3 and run4) the experiments at the LHC should be able to answer the above questions.

\section{References}

[1] P. Braun-Munzinger et al., Phys. Rep. 621 (2016) 76-126.

[2] R. Bala et al., Int. J. Mod. Phys. E 25 (2016) 1642006.

[3] U. Heinz, O. Evdokimov and P. Jacobs (editors), Nucl Phys. A 967 (2017) 1-1010.

[4] J.-Y. Ollitrault, Phys. Rev. D 46 (1992) 229

S.A. Voloshin and Y. Zhang, Z. Phys. C 70 (1996) 665

[5] B. Abelev et al. (ALICE Collaboration), Eur. Phys. J. C 74 (2014) 3054.

[6] J. Adam et al. (ALICE Collaboration), J. High Energ. Phys. (2015) 205.

[7] W. Adam et al. (CMS Collaboration), Phys. Lett. B 782 (2018) 474.

[8] S. Acharya et al. (ALICE Collaboration), Phys. Rev. Lett. 120 (2018) 102301.

[9] A.M. Sirunyan et al. (CMS Collaboration), Phys. Rev. Lett. 120 (2018) 202301.

[10] S. Acharya et al. (ALICE Collaboration), arXiv:1804.09083 submitted to J. High Energ. Phys.

[11] B. Abelev (ALICE Collaboration) Phys. Rev. Lett. 109 (2012) 072301.

[12] S. Chatrchyan et al. (CMS Collaboration) Phys. Rev. Lett. 109, 222301.

[13] S. Acharya et al. (ALICE Collaboration), Phys. Lett. B 766 (2017) 212.

[14] A.M. Sirunyan et al. (CMS Collaboration), Phys. Rev. Lett. 120 (2018) 142301.

[15] S. Acharya et al. (ALICE Collaboration), Phys. Rev. Lett. 119 (2017) 242301.

[16] S. Chatrchyan et al. (CMS Collaboration) Phys. Lett. B 718 (2013) 795.

[17] J. Adam et al. (ALICE Collaboration), Phys. Lett. B 760 (2016) 720.

[18] S. Ahmad et al. (ALICE Collaboration), JHEP 06 (2016) 50.

[19] J. Adam et al. (ALICE Collaboration), Nature Physics 13 (2017) 535.

[20] A.M. Sirunyan et al. (CMS Collaboration) arXiv:1804.09767, submitted to Phys. Rev. Lett.

[21] The ATLAS Collaboration; ATLAS-CONF-2017-006.

[22] S.Acharya et al. (ALICE Collaboration), Phys. Lett. B 780 (2018) 7. 